\title{
Debris-Blocking Sensitivity of Piano Key Weirs under Reservoir-Type Approach Flow
}

\author{
Michael Pfister ${ }^{1}$; Damiano Capobianco²; Blake Tullis, M.ASCE ${ }^{3}$; and Anton J. Schleiss ${ }^{4}$
}

\begin{abstract}
The collection of floating woody debris at flow control structures, such as spillways and weirs, can potentially result in reduced discharge efficiency (higher upstream head for a given weir discharge). Compared to less hydraulically-efficient control structures, piano key weirs have higher discharge efficiency (lower upstream heads for a given discharge), which may increase the likelihood of woody debris collection. A systematic laboratory study was conducted to evaluate the interaction between various piano key weir geometries and woody debris types and sizes. The results of individual (noncumulative) debris tests indicated that floating debris blockage probability is highly influenced by trunk diameter and upstream head. The effects of debris accumulation on the upstream head varied with the value of the debrisfree reference upstream head condition. At lower upstream reference head values, the cumulative debris tests indicated a relative increase of the debris-associated upstream head of approximately $70 \%$; higher upstream reference head values produced upstream head increases limited to approximately 20\%. DOI: 10.1061/(ASCE)HY.1943-7900.0000780. ( 2013 American Society of Civil Engineers.
\end{abstract}

CE Database subject headings: Debris; Probability; Weirs; Control structures; Floods; Spillways.

Author keywords: Piano key weir; Woody debris; Blocking probability; Discharge capacity.

\section{Introduction}

Floating wooden debris (i.e., driftwood) is often transported by a river course during flood events. Flooding associated with intense rain events in relatively dry zones can wash out unstable trees or deadwood. Under extreme conditions, landslides or bank erosion may add entire trees to a river. Woody debris, often combined with urban waste, can cause serious problems at hydraulic structures such as weirs (Bezzola and Hegg 2007), bridge decks (Bezzola et al. 2002; Schmocker and Hager 2011) or bridge piers (Melville and Dongol 1992; Pagliara and Carnacina 2010). Near structures, debris can block and thereby reduce the open flow area for water passage, which leads to reduction in discharge capacity and increase of upstream flow depth. The blocking generated by woody debris at weirs and spillway inlets is of particular interest, as the latter may affect the safety of such structures. Namely, the maximum spilling capacity may be drastically reduced due to blocked gates and/or weir obstruction.

Small and irregular flow cross-sections where accelerations occur are often prone to debris blockage. Depending on the size of the woody debris and the weir geometry, non-linear weirs, such as

\footnotetext{
${ }^{1}$ Research and Teaching Associate, Laboratory of Hydraulic Constructions, Ecole Polytechnique Fédérale de Lausanne, CH-1015 Lausanne, Switzerland (corresponding author). E-mail: michael.pfister@epfl.ch

${ }^{2}$ M.Sc. Student, Univ. of Cassino, I-03043 Cassino, Italy. E-mail: capobianco.damiano@gmail.com

${ }^{3}$ Associate Professor, Utah State Univ., 8200 Old Main Hill, Logan, UT 84322. E-mail: blake.tullis@usu.edu

${ }^{4}$ Professor, Laboratory of Hydraulic Constructions, Ecole Polytechnique Fédérale de Lausanne, CH-1015 Lausanne, Switzerland. E-mail: anton.schleiss@epfl.ch

Note. This manuscript was submitted on February 25, 2013; approved on May 22, 2013; published online on October 15, 2013. Discussion period open until April 1, 2014; separate discussions must be submitted for individual papers. This paper is part of the Journal of Hydraulic Engineering, Vol. 139, No. 11, November 1, 2013. C ASCE, ISSN 0733-9429/2013/11$1134-1141 / \$ 25.00$.
}

piano key weirs (PKWs), can also function as debris collectors. The nonlinear nature of PKWs (folded back and forth in plan view to make repeating cycles or keys) allows for significantly longer weir lengths than could be achieved using a traditional linear weir for a given spillway channel width. The narrow design of the PKW's inlet and outlet keys produce an increased potential for debris collection (e.g., the weir functions similarly to a trash rack for longer woody debris). The high discharge efficiency of PKWs relative to linear weirs can also influence debris collection; the flow depth over a PKW for a given discharge will be less than would be required by linear structures, thus providing less flow momentum per unit of weir length for flushing debris. If the catchment is forested, the potential effects of driftwood on the PKW head-discharge relation should be considered as part of the hydraulic design (Leite Ribeiro et al. 2012a, b).

There are few studies published on the effect of debris at PKWs. Ouamane and Lempérière (2006) conducted preliminary model tests with floating debris. They observed that no debris was collected under the apex overhangs during the reservoir filling process. During PKW operation, however, debris got stuck in the inlet key at small discharges, resulting in a slight reduction in discharge efficiency. A discharge coefficient reduction of about $10 \%$ was reported for $H / P<0.5$, where $H=$ total upstream head measured relative to the $\mathrm{PKW}$ crest elevation, and $P=$ weir height. As the weir's discharge increased, the debris was eventually washed downstream. In 2007, the Laboratory of Hydraulic Constructions (LCH) investigated [Barrage de Saint-Marc: Etude sur modèle des évacuateurs de cure (Saint-Marc Dam: Spillway model study), unpublished report] the effects of floating wood on the St. Marc Dam (France) emergency PKW spillway and reported no debrisassociated reduction in discharge capacity when the gates of the adjacent principal spillway were fully open, attracting a significant portion of the debris. Laugier (2007) reports of systematic model tests with debris of the emergency PKW spillway of Goulours Dam (France), concluding that (1) an increasing head tends to eventually remove previously blocked debris; (2) the principal gated spillways attracts debris before the PKW starts operation; (3) most debris 


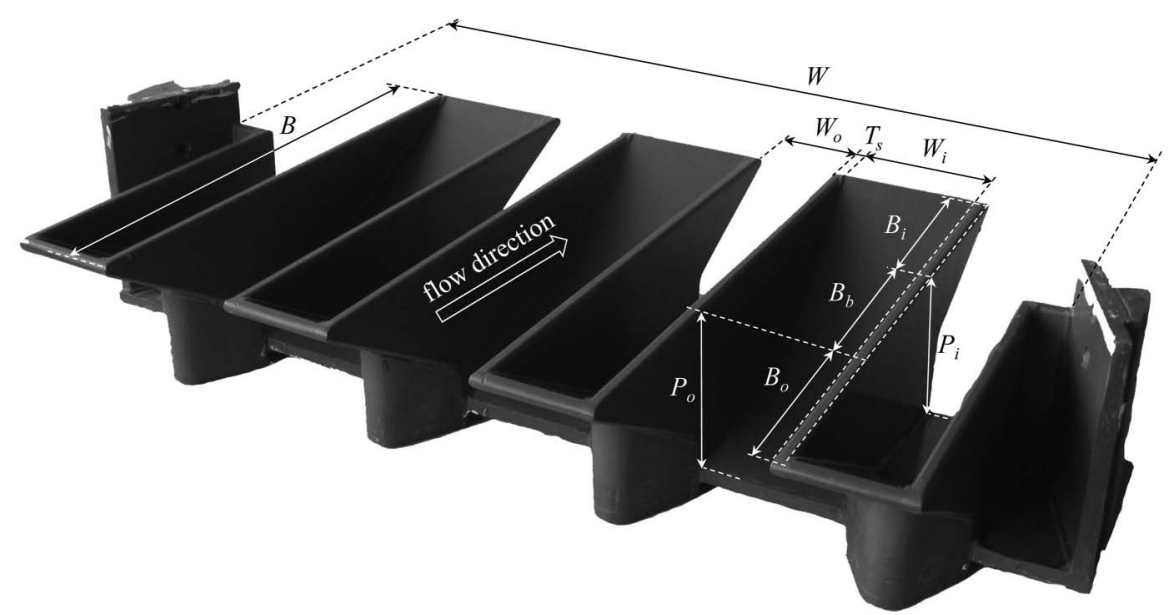

Fig. 1. Notation of PKW

passes the PKW for flow depths at the PKW larger than $1 \mathrm{~m}$; (4) most streamlines pass below the debris of a fully blocked PKW entering the inlet key (i.e., water flows around the blockage); and (5) the residual discharge of a blocked PKW is more than $80 \%$ of the debris-free condition. Sogreah, France [Modèle physique du barrage de Luzieres (Physical model of the Luzieres Dam), unpublished report] conducted model tests of the Luziere Dam (France) emergency PKW spillway. They observed that (1) debris was mainly attracted by the principal spillway inlet, as it generated higher approach flow velocities than the PKW; (2) the volume of blocked debris decreased with increasing discharge; (3) the debris accumulations were compact for high heads, and loose for small heads; and (4) a relative head increase of some $10 \%$ occurred for the 100-year flood, with an initial debris-free upstream reference head of $1 \mathrm{~m}$.

The present paper describes systematic model testing of the effect of debris on the PKW discharge efficiency in the absence of additional spillway structures (e.g., gated spillways) operating in parallel (i.e., all floating debris interacts with the PKW). The effect of wooden trunks and rootstocks on the discharge efficiency of three different laboratory-scaled PKW geometries was investigated. The first test series determined the blocking probability of individual debris elements, indicating the critical trunk and rootstock sizes associated with the onset of debris blockage (at specific weir discharges) for three different PKW geometries. The second test series more closely approximated real-world conditions by investigating the nature of driftwood element accumulation on the PKWs and the effect on discharge efficiency. Herein, the standard geometric notation for PKWs (Fig. 1) is applied (Pralong et al. 2011), where $B=$ streamwise length, $R=$ parapet wall height, $T_{s}=$ side wall thickness, and $W=$ transverse width. Furthermore, subscript $i$ refers to the inlet key, i.e., the key that is filled with water when the reservoir water surface equals the PKW crest elevation; and subscript $o$ refers to the outlet key. These subscripts may be used in the context of $B, P$, and $W$ (Fig. 1).

\section{Experimental Setup}

\section{Physical Models}

Systematic physical model tests were conducted at the LCH of the Ecole Polytechnique Fédérale de Lausanne (EPFL), Switzerland. The PKWs were inserted at the end of a 2-m-wide and approximately 10-m-long channel, with an approach flow depth on the order of $0.8 \mathrm{~m}$ (Fig. 2). The PKWs were installed in the opening of a vertical-walled dam (contracted-weir configuration) as shown in Figs. 2 and 3. With the $W$ to upstream channel width ratios ranging from 0.27 to 0.33 and the base of each PKW installed around $0.7 \mathrm{~m}$ above the channel invert, the upstream approach flow condition approximated that of a reservoir, and all test conditions were free of tailwater submergence effects. The water level in the channel was measured using a point gauge $( \pm 0.5 \mathrm{~mm})$. The latter measurement provided $H$ (total upstream head), as the measurement was taken in a zone with stagnant water. Generally, the channel flow velocities tended to zero because the tested discharges were small. The discharge $Q$ was measured with a magnetic inductive flow meter $( \pm 0.5 \%$ at full span).

Relatively limited driftwood blockage occurred for values of $H$ exceeding the frequently-applied minimum $H$ limit of $0.03 \mathrm{~m}$ regarding discharge capacity scale effects (Novak et al. 2010). However, as the head-discharge relations were not the primary focus of this investigation and because all $H$ comparisons were based on relative head differences, the potential influences of viscosity and surface tension size-scale effects were not taken into account. In an effort to better generalize the results, three different Type-A [including upstream and downstream overhangs; see Lempérière et al. (2011) for definition of other types] PKW configurations

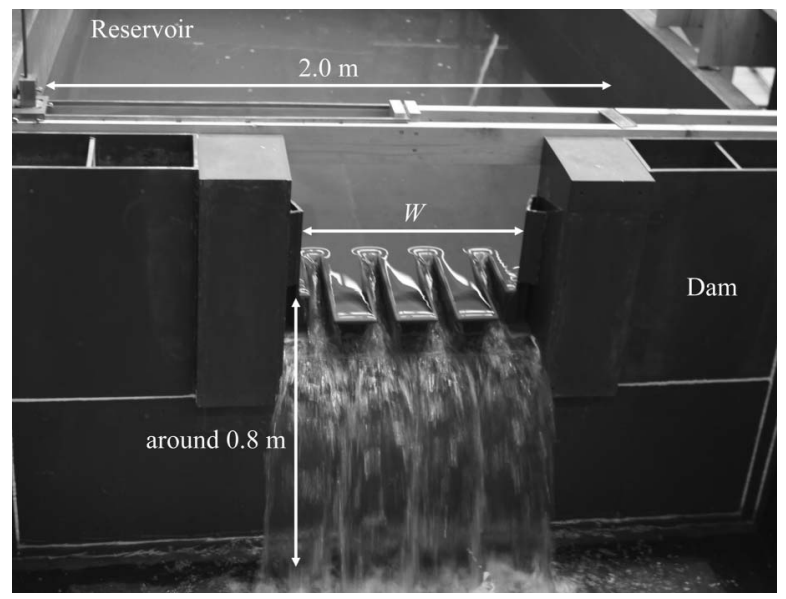

Fig. 2. Experimental set-up, with configuration A 


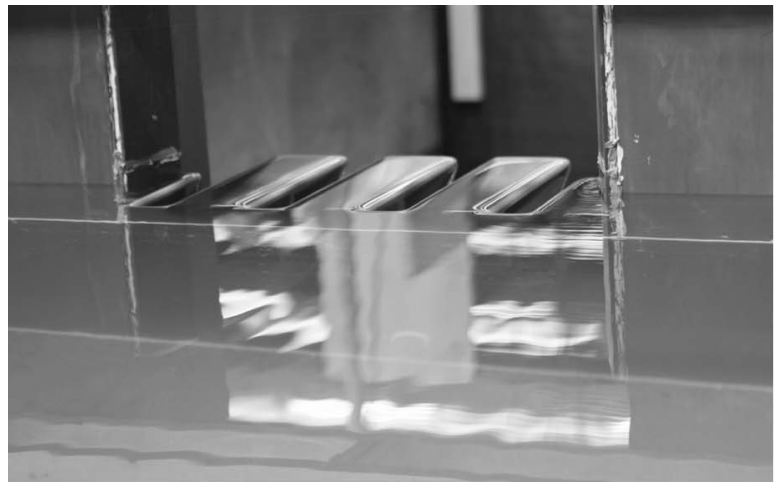

(a)

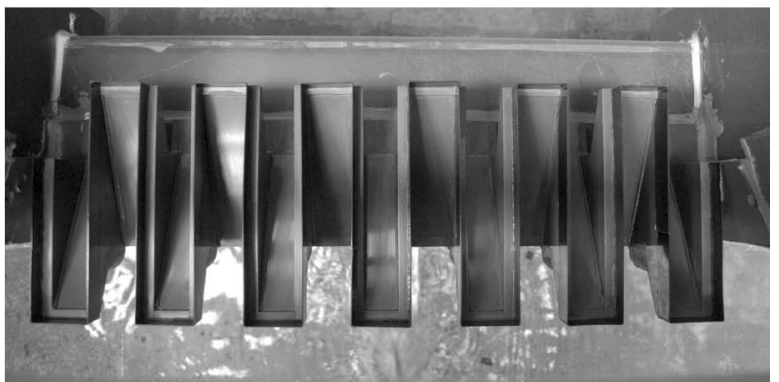

(b)

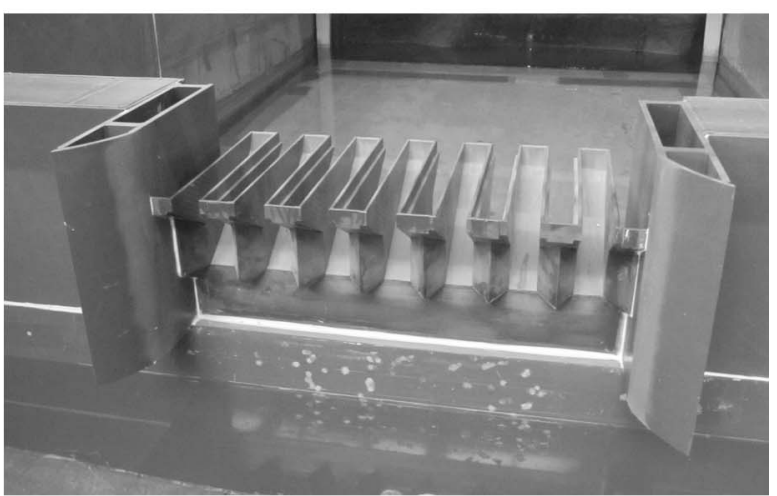

(c)

Fig. 3. Photos of tested PKWs, configurations (a) A; (b) B; and (c) C

were tested as detailed in Table 1 and Fig. 3, which included variations in cycle number and characteristic length. The abutments of the configurations $\mathrm{A}$ and $\mathrm{B}$ were abrupt (including a short oblique connection wall between dam and PKW), whereas configuration $\mathrm{C}$ was a rounded wing-wall reaching into the reservoir (Fig. 3).

\section{Debris}

Floods often carry driftwood into a watercourse. The size and shape of the driftwood elements are also influenced by the gradient of the river, discharge rates, and the size and quantity of the rocks, cobbles, and/or boulders lining the channel bed. Consequently, driftwood can be characterized by a large variety of dimensions. Beyond the man-made debris elements, natural debris in steepergradient river channels consists mostly of trunks and rootstocks (i.e., floating trees impacting on boulders typically broken up into smaller individual branch, trunk, and rootstock elements).

The herein-defined wood distribution was based on two approaches, namely (1) a large spectrum of trunk lengths to find the sensitive dimensions; and (2) common flood-related debris element sizes as determined by Bezzola and Hegg (2007). For practical reasons, the trunks were sorted into seven size classes, as given
Table 1. Configurations of Tested PKWs A, B, and C (Model Dimensions)

\begin{tabular}{lcccc}
\hline Configuration & A & B & C & Unit \\
\hline$W$ & 0.545 & 0.555 & 0.665 & {$[\mathrm{~m}]$} \\
$W_{i}$ & 0.082 & 0.044 & 0.040 & {$[\mathrm{~m}]$} \\
$W_{o}$ & 0.050 & 0.039 & 0.033 & {$[\mathrm{~m}]$} \\
$W_{u}$ & 0.148 & 0.085 & 0.093 & {$[\mathrm{~m}]$} \\
$T_{s}$ & 0.008 & 0.001 & 0.010 & {$[\mathrm{~m}]$} \\
$B$ & 0.325 & 0.190 & 0.325 & {$[\mathrm{~m}]$} \\
$B_{o}$ & 0.118 & 0.055 & 0.100 & {$[\mathrm{~m}]$} \\
$B_{i}$ & 0.090 & 0.050 & 0.075 & {$[\mathrm{~m}]$} \\
$P_{i}=P_{o}$ & 0.096 & 0.084 & 0.150 & {$[\mathrm{~m}]$} \\
$R$ & 0.000 & 0.017 & 0.020 & {$[\mathrm{~m}]$} \\
$L / W$ & 5.20 & 5.50 & 7.80 & {$[-]$} \\
$W_{i} / W_{o}$ & 1.64 & 1.13 & 1.21 & {$[-]$} \\
$B / P$ & 3.39 & 2.26 & 2.17 & {$[-]$} \\
$B_{i} / B$ & 0.28 & 0.26 & 0.23 & {$[-]$} \\
$B_{o} / B$ & 0.36 & 0.29 & 0.31 & {$[-]$} \\
\hline
\end{tabular}

Table 2. Trunk Characteristics (Model Dimensions)

\begin{tabular}{lccccccc}
\hline $\begin{array}{l}\text { Size } \\
\text { class } c\end{array}$ & 1 & 2 & 3 & 4 & 5 & 6 & 7 \\
\hline$T[\mathrm{~m}]$ & 0.033 & 0.100 & 0.167 & 0.233 & 0.300 & 0.367 & 0.433 \\
$D[\mathrm{~m}]$ & 0.002 & 0.005 & 0.008 & 0.012 & 0.015 & 0.018 & 0.022 \\
\hline
\end{tabular}

in Table 2 and Fig. 4(a), where $T=$ trunk length and $D=$ trunk diameter. Rootstock testing was limited to a single set of similarly sized samples [Fig. 4(b)]; the variability of some characteristic dimensions is presented in Table 3 . They include $T, D$, and the effective diameter $U$ of the root spread, measured in the plane perpendicular to the trunk axis. The dimensionless trunk ratios $T / W_{u}$ and $D / W_{u}$ (Fig. 5), where $W_{u}$ represents the cycle width (i.e., $W_{u}=W_{o}+W_{i}+2 T_{s}$ ), can be used to link the relative size of the debris (Tables 2 and 3 ) to the PKW model dimensions (Table 1).

Two test series were conducted.

1. Individual trunk and rootstock test: At various discharge conditions, 25 to 50 trunks of every size class (Table 2) were added individually at random stations across a common reservoir cross-section located approximately $2 \mathrm{~m}$ upstream of the PKW and the outcome noted (trapped or passed). If an individual trunk got caught on the weir, it was removed before the next trunk addition upstream, intentionally avoiding debris accumulation effects.

2. Accumulative debris test: Ten different debris batches were prepared, featuring 19 to 22 trunk elements of varied sizes and two rootstocks, as detailed in Table 4. Further, the trunk dimensions within a debris batch were chosen in an effort to be consistent with the natural driftwood debris size distribution statistics presented by Bezzola and Hegg (2007). The 10 debris batches were added sequentially at random stations across a common reservoir cross-section located $2 \mathrm{~m}$ upstream of the PKW, for a constant $Q$. After each individual debris batch addition, the number and sizes of the passing and trapped elements were noted along with the corresponding $H$. Without removing the debris trapped on the PKW from previous batches, subsequent debris batches were introduced upstream of the flume; this was repeated for all 10 debris batches. All three PKW configurations (Table 1) were tested under four different discharge rates (discharge was held constant during a test) spanning a dimensionless upstream head spectrum of $0.07 \leq H_{r} / P \leq 0.49$, where $H_{r}=$ reference upstream $H$ prior to adding debris. For each discharge, the 10 batches were added sequentially and their effect on $H$ measured. Thus, 


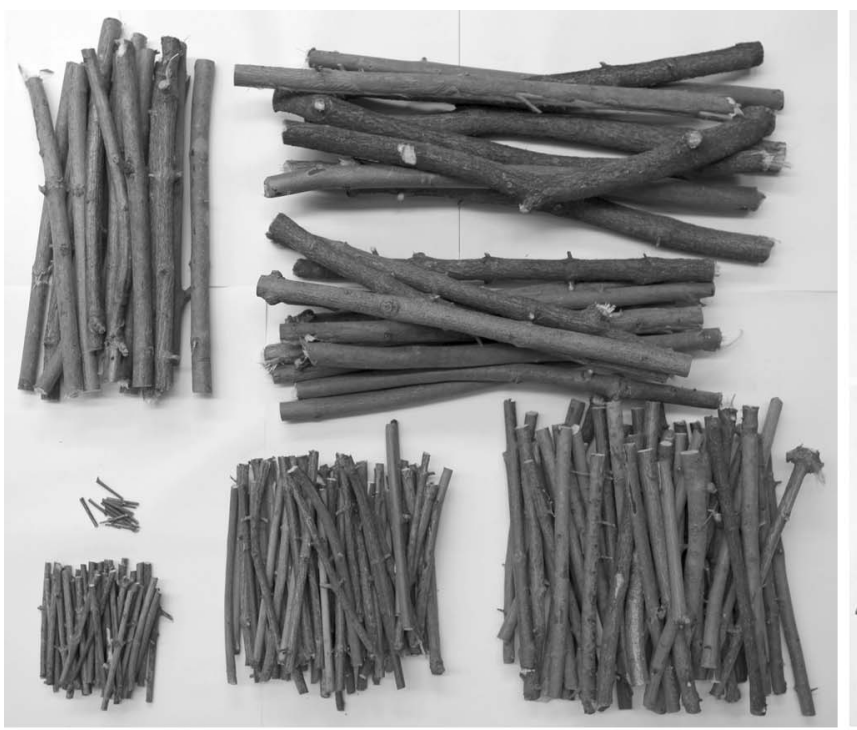

(a)

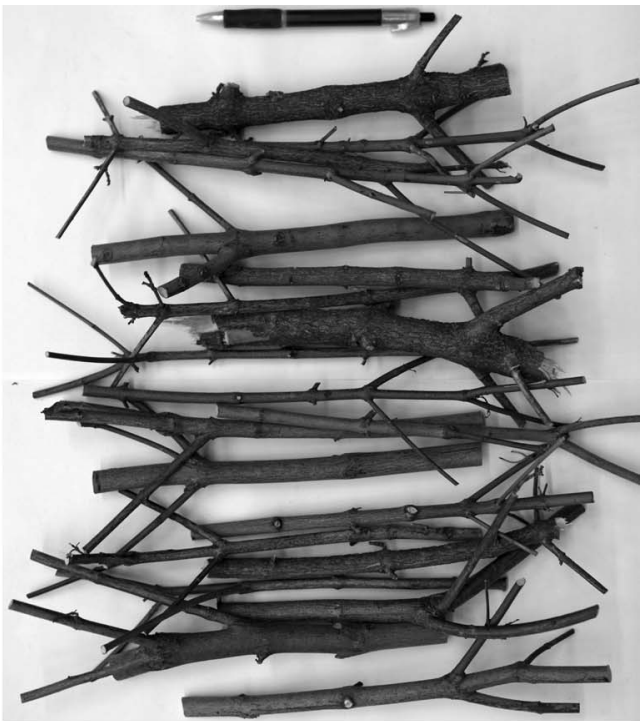

(b)

Fig. 4. Model debris including (a) trunks (size classes according to Table 2); and (b) rootstocks (Table 3)

Table 3. Rootstock (RS) Characteristics (Model Dimensions)

\begin{tabular}{lccc}
\hline Dimension & $T[\mathrm{~m}]$ & $D[\mathrm{~m}]$ & $U[\mathrm{~m}]$ \\
\hline Average & 0.160 & 0.009 & 0.088 \\
Minimum & 0.130 & 0.006 & 0.073 \\
Maximum & 0.188 & 0.018 & 0.105 \\
Standard deviation & 0.015 & 0.004 & 0.010 \\
\hline
\end{tabular}

40 values of $H$ resulted per PKW geometry, giving a total of 120 data points for all PKWs collectively.

\section{Blocking Probability for Individuals}

\section{General Behavior}

In the laboratory tests, the trunks' longitudinal axis typically selfaligned to the approach flow streamlines (i.e., parallel to the channel centerline) before touching the PKW crest, independent of the initial orientation with which they were introduced to the reservoir flow. Trunks arriving individually at the PKW show a typical behavior, depending on $T$ and $H$.

- Relatively short trunks under relatively high $H$ pass the PKW independent of location and initial orientation;

- The results for midsized trunks under medium $H$ were as follows: trunks oriented parallel to the streamlines entering a PKW inlet key passed while trunks contacting the upstream apex of the outlet key rarely did;

- For long trunks and low $H$, the probability of blockage increased significantly. Often, a rotation of the trunks from a parallel to a perpendicular orientation (relative to the approach flow streamlines) was observed, as soon as the leading end of the trunk made contact with the PKW outlet key apex. These trunks would always be trapped, whereas trunks orientated parallel to the streamlines passed with a higher probability.

Rootstocks were more sensitive and began to be blocked at lower $H$ than the trunks. For relatively small $H$, they pass if arriving at the PKW orientated perpendicular to the flow with the roots near an outlet key. Otherwise, particularly if entering an inlet key, they were typically trapped. For large $H$, they normally passed independent of location and orientation.
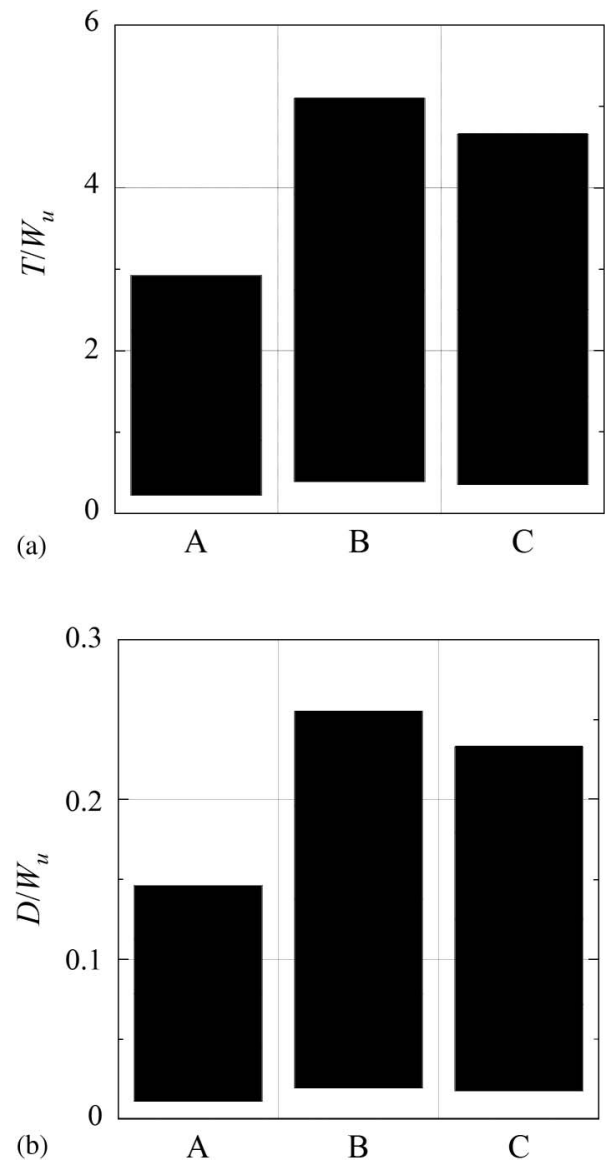

Fig. 5. Trunk (a) length; and (b) diameter, both normalized with cycle width, for configurations $\mathrm{A}, \mathrm{B}$, and $\mathrm{C}$

\section{Debris-Trapping Probability}

A data analysis of the individual trunk and rootstock tests determined that both $D$ and $H$ significantly influence the blocking probability ( $\Pi$ ) of individual trunks or rootstocks. Here, $\Pi$ is defined as the ratio of trapped elements to the number of supplied elements. 
Table 4. Debris Batch Size Characteristics, Giving Number $n$ of Individual Elements per Batch

\begin{tabular}{|c|c|c|c|c|c|c|c|c|c|c|}
\hline \multirow{3}{*}{$\begin{array}{l}\text { Batch } \\
\text { number } m\end{array}$} & \multicolumn{7}{|c|}{ Number of trunk } & \multirow{3}{*}{$\begin{array}{c}\text { Number of } \\
\text { rootstock }\end{array}$} & \multirow{3}{*}{$\begin{array}{l}\text { Elements } \\
\text { per batch }\end{array}$} & \multirow[b]{3}{*}{$V\left(10^{-3} \mathrm{~m}^{3}\right)$} \\
\hline & \multicolumn{7}{|c|}{ Size class $c$} & & & \\
\hline & 1 & 2 & 3 & 4 & 5 & 6 & 7 & & & \\
\hline 1 & 1 & 2 & 2 & 4 & 7 & 4 & 2 & 2 & 24 & 0.63 \\
\hline 2 & 1 & 1 & 1 & 4 & 6 & 4 & 2 & 2 & 21 & 1.10 \\
\hline 3 & 1 & 1 & 2 & 5 & 7 & 5 & 0 & 2 & 23 & 1.66 \\
\hline 4 & 1 & 1 & 1 & 4 & 6 & 4 & 2 & 2 & 21 & 2.14 \\
\hline 5 & 1 & 1 & 2 & 5 & 7 & 4 & 2 & 2 & 24 & 2.70 \\
\hline 6 & 1 & 1 & 1 & 5 & 7 & 5 & 0 & 2 & 22 & 3.20 \\
\hline 7 & 1 & 1 & 2 & 4 & 6 & 4 & 2 & 2 & 22 & 3.73 \\
\hline 8 & 1 & 1 & 1 & 5 & 7 & 4 & 2 & 2 & 23 & 4.23 \\
\hline 9 & 0 & 1 & 2 & 5 & 7 & 4 & 0 & 2 & 21 & 4.63 \\
\hline 10 & 0 & 2 & 2 & 5 & 6 & 4 & 2 & 2 & 23 & 5.12 \\
\hline
\end{tabular}

Though also important, the significance of $T$ on $\Pi$ was minor. The individual trunk data, presented in Fig. 6(a), show that the probability of trunk blockage is high (i.e., $\Pi=1$ ) when the trunk diameter is greater than $H$ (i.e., $D / H>1$ ). When $H \cong D$, the flow momentum was generally insufficient to carry the trunks over the PKW. The effective flow depth at the weir crest will be less than $H$ due to local flow accelerations. Trunks consistently passed $(\Pi=0)$ when $D / H<0.3$. For intermediate $D / H$, the blocking probability may be expressed pragmatically as

$$
\Pi=1.5\left(\frac{D}{H}\right)-0.5 \text { for } 0.33<D / H<1.00
$$
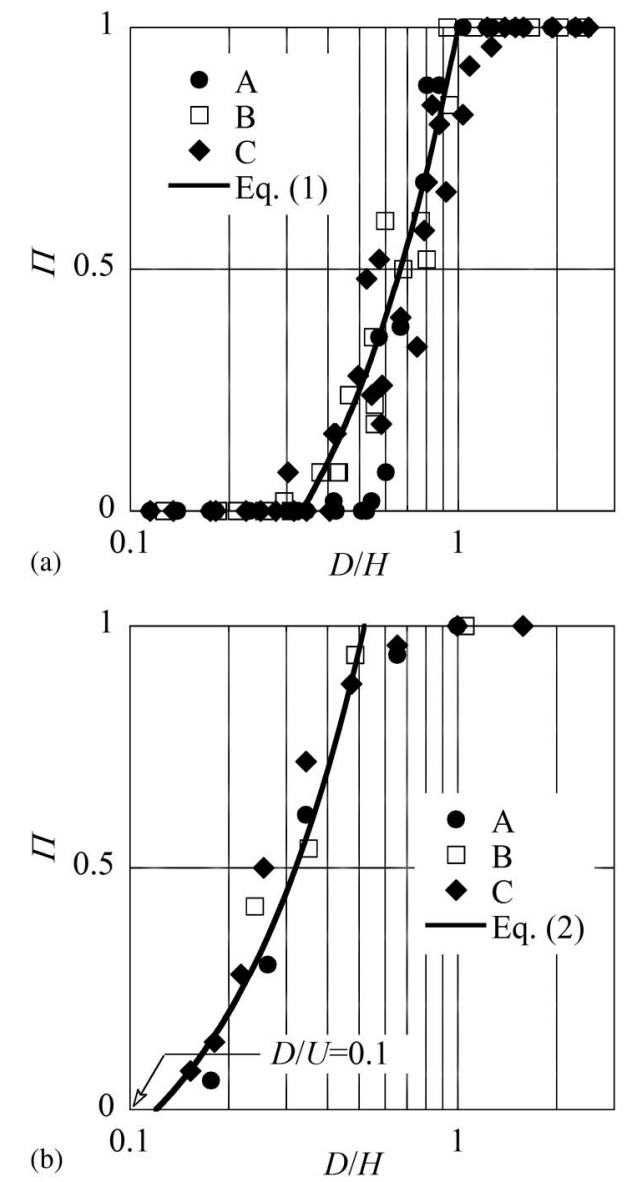

Fig. 6. Blocking probability $\Pi$ versus $D / H$ for (a) trunks; and (b) rootstocks
According to Eq. (1), a blocking probability of $\Pi=0.5$ corresponds to $D=(2 / 3) H$, a relationship analogous to critical flow depth. Machiels et al. (2011) observed a critical flow section near the downstream apex of the inlet key for small values of $Q$.

As shown in Fig. 6(b), the blocking probabilities of rootstocks are, as expected, higher than those of trunks with respect to $D / H$. With few exceptions, rootstocks blockage occurred when $D / H>$ 0.5 . Comparatively, $\Pi=0.25$ for trunk blockage when $D / H=$ 0.5 . Rootstock passage occurred $(\Pi=0)$ at approximately $D / H<0.1$. The average $D / U$ was 0.1 for the rootstocks tested, which was consistent with the $D / H$ for $\Pi=0$ value. This means that rootstocks will generally pass a PKW as long as the effective root diameter $U$ is equal or smaller than $H$. For intermediate $D / H$, the blocking probability may be expressed pragmatically as

$$
\Pi=2.5\left(\frac{D}{H}\right)-0.3 \text { for } 0.12<D / H<0.52
$$

According to Eq. (2) a blocking probability of $\Pi=0.5$ results if $D=(1 / 3) H$, i.e., $D$ is equal to half the critical flow depth. For both, Figs. 6(a and b), no significant difference for the three tested PKW configurations (Table 1) is recognizable.

\section{Effect of Debris Accumulations}

\section{General Behavior}

The accumulative effects of debris were evaluated for a range of $D$ and $H_{r}$ values $\left(0.03 \leq D / H_{r} \leq 3.62\right)$. As seen in the individual element tests, the probability of debris blockage increases with decreasing $H$ (and $Q$ ). The blocked trunks typically orientated themselves perpendicular to the PKW crest, whereas the trunks accumulated upstream in the reservoir were of arbitrary orientated. For large $H$ (and $Q$ ) values, most debris passed the PKW and the blocked elements generated only a small increase of $H$.

Although the normalization $H_{r} / P$ seems inadequate to fully describe the behavior of debris, the tests indicated that the maximum observed relative head increase $\left(H / H_{r}\right)$ was $70 \%$ for $0.07 \leq H_{r} /$ $P \leq 0.2$, and below $20 \%$ for $0.2 \leq H_{r} / P \leq 0.5$ [Fig. 7(a)]. The relative effect of the debris on $H$ decreases with increasing $H_{r}$. The data in Fig. 7(a) show that $H / H_{r}$ effects are more influenced by $H_{r} / P$ variation than the supplied debris volume $m(m$ represents the incremental number of debris batches introduced into the system, which ranged from 1 to 10 for each test). The $H / H_{r}$ response to $m=5$ and $m=10$ were essentially the same for values of $H_{r} / P>0.2$ in Fig. 7(a). This suggests that for $H_{r} / P>0.2$, above a certain limit for floating debris volume, the addition of more floating debris will have little effect on the weir's discharge efficiency.

The relationship between $\Pi$ and $H / H_{r}$ for the accumulative debris test is given in Fig. 7(b). For $\Pi<0.8$ the value $H / H_{r}$ marginally increases $\left(H / H_{r} \cong 1.1\right)$. For a complete blocking $(0.8<\Pi<1.0)$, as observed herein for small $H$ and high debris volumes, the relative head significantly increases up to $H / H_{r} \cong$ 1.7. The first trunks only slightly increase the relative head, whereas complete blocking of most elements is required to significantly affect $H$.

Fig. 8 shows photographic examples of the debris accumulation tests for each of the three PKW geometries tested following the addition of the 10 driftwood batches. It indicates that the blockage is a function of $H$ rather than of $H_{r} / P$ : A pronounced blockage is visible for the smallest head [Fig. 8(a)] and minimal blockage at the largest head [Fig. 8(c)]. 

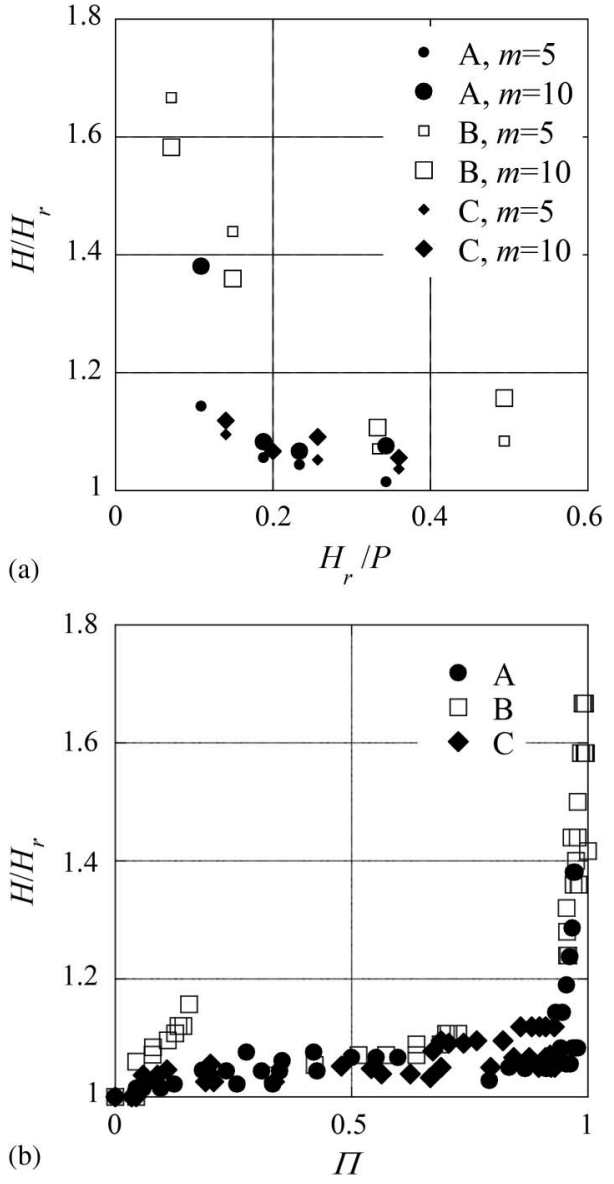

Fig. 7. Relative head increase $H / H_{r}$ as a function of (a) $H_{r} / P$; and (b) $\Pi$

\section{Effect of Debris Volume}

For practical use, the estimation of the head variation and the debris trapping probability are of interest, both as a function of $H$ and the debris volume expected at a PKW. In contrast to the individual tests, the trunk diameter $D$ could not be considered as reference herein, as the accumulations included the entire range of $D$.

The progressively added debris volume $V$ (Table 4 ) is defined as

$$
V=\sum_{c, m}\left(n \frac{D_{c}^{2} \pi}{4} T\right)
$$

Here, $c$ denotes the individual trunk class (Table 2), and $n=$ number of trunks and rootstocks per class (Table 4). Thus, $V$ is the integral volume of the debris arriving at the PKW for a certain event, starting with $V=0$ before debris occurrence and then continuously increasing with the number of considered batches (Table 4).

In an effort to normalize the debris volume $V$ with a reference hydraulic volume, several alternatives were evaluated. The data analysis indicated that the normalization shall include $H_{r}$, because the blocking process and its effect on $H$ are particularly a function of the latter. To further express $V$ in specific terms, the weir width $W$ was considered; the reference volume was selected as $H_{r}^{2} W$. Fig. 9 shows $\Pi$ and $H / H_{r}$ as a function of $V /\left(H_{r}^{2} W\right)$. With increasing $V$, the values of $\Pi$ and $H / H_{r}$ both increase. As visible in Fig. 9(a), the blocking process starts at small $V /\left(H_{r}^{2} W\right)$. Complete blockage of the debris elements $(\Pi=1)$ approximately occurs

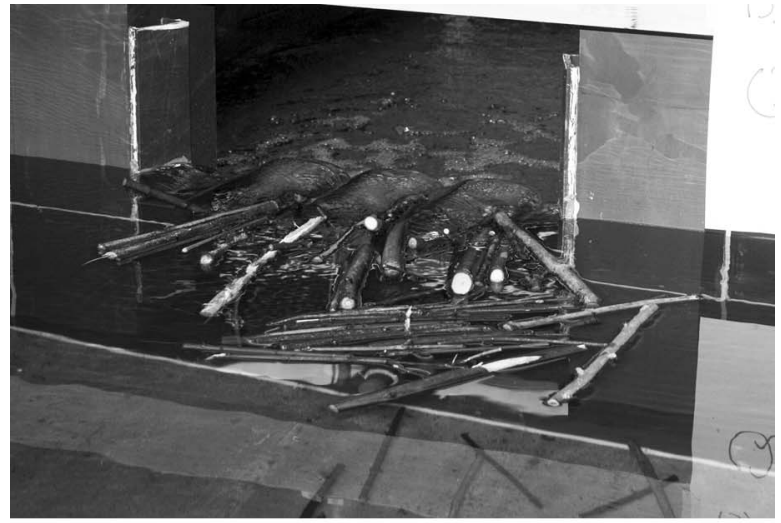

(a)

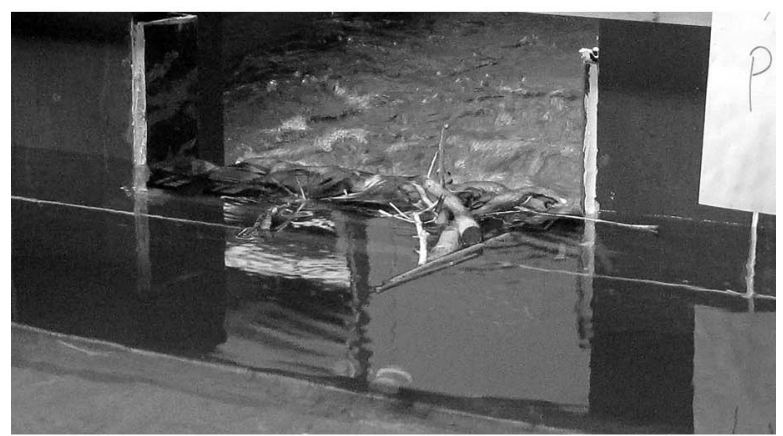

(b)

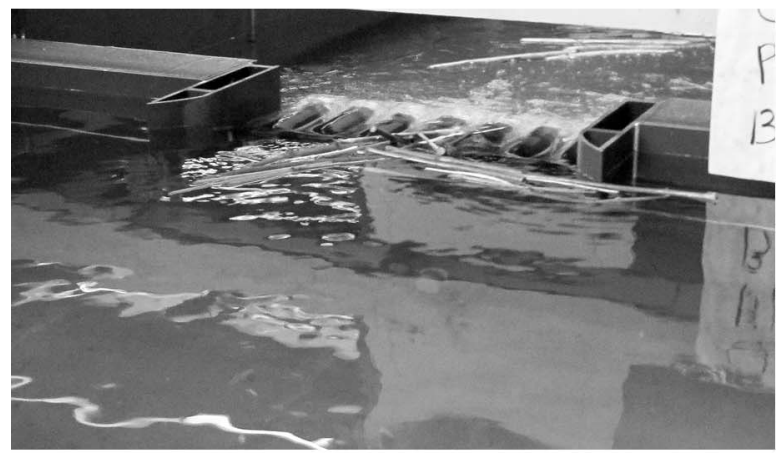

(c)

Fig. 8. Blockage situation following the addition of 10 debris batches, for configurations (a) A $\left(H=0.033 \mathrm{~m}, H_{r} / P=0.34\right)$; (b) $\mathrm{B}\left(H=0.042 \mathrm{~m}, H_{r} / P=0.49\right)$; and (c) $\mathrm{C}(H=0.054 \mathrm{~m}$, $\left.H_{r} / P=0.36\right)$

when $V=(20$ to 30$)\left(H_{r}^{2} W\right)$. As for the effect of $V /\left(H_{r}^{2} W\right)$ on $H / H_{r}$, Fig. 9(b) shows that it remains roughly below $10 \%$ for $V<15\left(H_{r}^{2} W\right)$. For extensive debris volumes (e.g., $100\left(H_{r}^{2} W\right)$ ), $H$ increases by approximately $60 \%$; however, a pronounced data scatter is visible. Since the design capacity of a PKW typically includes comparatively large values $H_{r}$, the relative effect of the debris on $H / H_{r}$ is small.

The effects of $V /\left(H_{r}^{2} W\right)$ on $\Pi$ and $H / H_{r}$ may be approximated by

$$
\Pi \cong \tanh \left(0.150 \frac{V}{H_{r}^{2} W}\right) \text { for } 0<V /\left(H^{2} W\right)<100
$$

and

$$
\frac{H}{H_{r}} \cong 1+\tanh \left(0.007 \frac{V}{H_{r}^{2} W}\right) \quad \text { for } 0<V /\left(H^{2} W\right)<100
$$



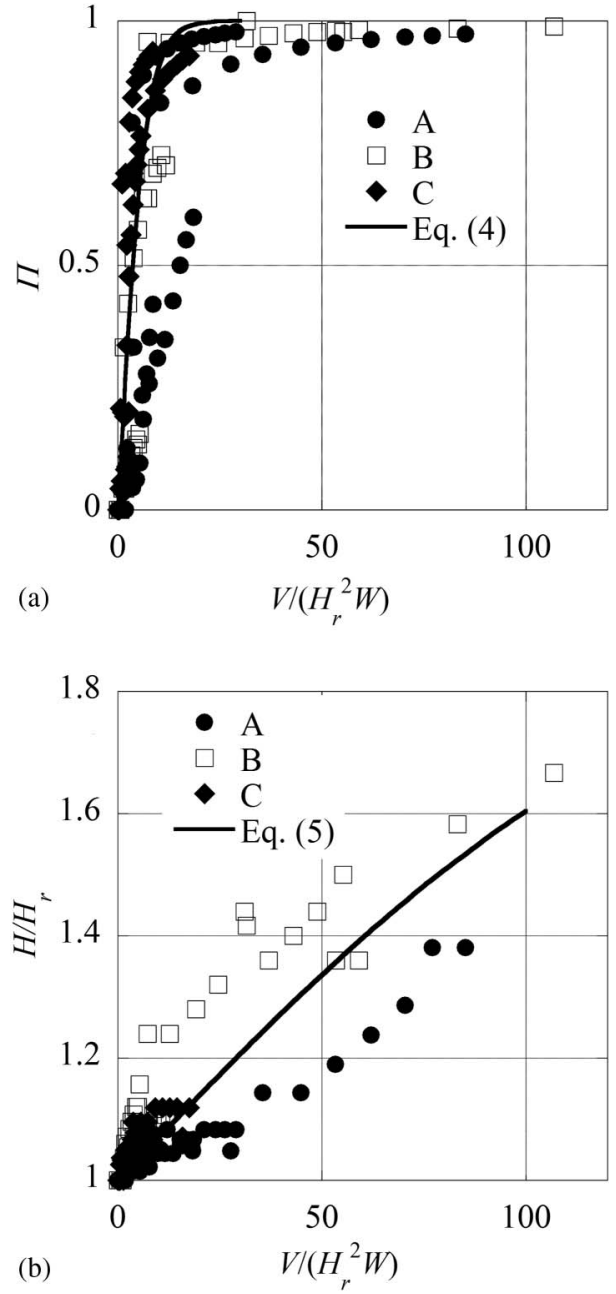

Fig. 9. Effect of $V /\left(H_{r}^{2} W\right)$ on (a) $\Pi$; and (b) $H / H_{r}$

\section{Conclusions}

Floating debris includes a wide range of objects and sizes. Consequently, a large variety of trunk and rootstock geometries were tested. Though hopefully somewhat representative of prototype debris elements, the results presented herein apply to the specific debris conditions, PKW weir, and abutment wall geometries investigated. Besides the characteristics of the individual debris elements, occurrences of driftwood upstream of the PKW may vary from individual elements to large accumulations. The results presented herein give trends to the effect of debris at PKWs.

The dimensionless debris diameter $(D / H)$ was found to be the dominant parameter indicating whether an individual trunk will be trapped by or pass over a PKW. A 50\% blocking probability was observed if the driftwood diameter equaled the PKW critical flow depth (i.e., 2/3H). Trunk length, though relevant was less significant. The debris elements used in this study featured lengths that were proportional to their diameter $(T / D=20)$. For accumulations, the data indicated a less homogenous trend. In general, an increase in head is recognizable with increasing debris volume collecting at the PKW. This trend is less pronounced for large debris volumes combined with a significant reference head $\left(H_{r} / P>0.2\right)$. It is essential that the relative increase of the head is only significant when the reference (before debris occurrence) $H$ is small, i.e., for smaller discharges. For large heads, typically used as PKW design values, the relative increase of the head reduces, as some debris is washed over the crest by the increasing hydraulic load.

In this study, the PKW approach flow condition was limited to a reservoir-type. The effect of floating debris on the PKW head-discharge relationship may vary with alternative PKW approach flow conditions (e.g., channelized approach with non-negligible approach flow velocities). Based on the results of this study, the following conclusions are made:

1. For individual trunk debris, the principle dimensional parameters influencing the probability of debris blockage (П) are the trunk diameter $(D)$ and the upstream head $(H)$. The trunk length had a secondary influence;

2. The trunk blockage probability was $100 \%(\Pi=1.0)$ when the trunk diameter to upstream head ratio $D / H \geq 1.0$. The trunks all passed $(\Pi=0)$ when $D / H \leq 0.3$;

3. As would be expected, the rootstocks were more sensitive to being blocked than trunks due to the larger root diameter. The rootstock blockage probability was $100 \%(\Pi=1.0)$ when the $D / H>0.5$ or the equivalent rootstock diameter to $H$ ratio $(U / H)>5.0$. The rootstocks all passed $(\Pi=0)$ when $D / H<$ 0.1 or $U / H<1.0$;

4. For low heads $\left(H_{r} / P<0.2\right)$, the debris accumulations increased $H$ by as much as $70 \%$. For higher heads $\left(0.2<H_{r} / P<5.0\right)$, the debris accumulation-induced increase in $H$ was below $20 \%$. For $H_{r} / P>0.2$, above a certain debris volume limit, the addition of more floating debris resulted in a marginal increase in $H$;

5. With the product of the square of the upstream head and channel width $\left(H_{r}^{2} W\right)$ representing a reference hydraulic volume with respect to debris blockage, a debris volume $V=$ $15 H_{r}^{2} W$ increased $H$ by $10 \%$. Finally, $\Pi=1.0$ (100\% blockage) when $V=(20$ to 30$) H_{r}^{2} W$, and $H / H_{r} \cong 1.6$ when $V=100 H_{r}^{2} W$;

6. A priori, the PKW configuration and the abutment wall details seem of minor relevance regarding the sensitivity of debris accumulation and its effect on the head increase.

\section{Notation}

The following symbols are used in this paper:

$B=$ streamwise length [m];

$B_{b}=$ base length [m];

$c=$ trunk size class;

$D=$ trunk diameter $[\mathrm{m}]$;

$H=$ total approach flow head [m];

$L=$ developed crest length [m];

$m=$ batch number, 1 to $10[-]$;

$n=$ number of trunks and rootstocks per class [-];

$P=$ vertical height $[\mathrm{m}]$;

$Q=$ discharge $\left[\mathrm{m}^{3} / \mathrm{s}\right]$;

$R=$ parapet wall height $[\mathrm{m}]$;

$T=$ trunk length [m];

$T_{s}=$ side wall thickness [m];

$U=$ rootstock diameter $[\mathrm{m}]$;

$V=$ cumulative supplied debris volume $\left[\mathrm{m}^{3}\right]$;

$W=$ transversal width [m]; and

$\Pi=$ blocking probability [-].

\section{Subscripts}

$i=$ inlet key;

$o=$ outlet key;

$r=$ reference, before start of debris addition; and

$u=$ cycle. 


\section{References}

Bezzola, G. R., Gantenbein, S., Hollenstein, R., and Minor, H.-E. (2002). "Verklausung von Brückenquerschnitten [Blocking of bridge cross sections]." VAW Mitteilung, H.-E. Minor, ed., Vol. 175, ETH Zurich, Switzerland, 87-97 (in German).

Bezzola, G. R., and Hegg, C., eds. (2007). Ereignisanalyse Hochwasser 2005 [2005 Flood event analysis], Bundesamt für Umwelt \& Eidgenössische Forschungsanstalt für Wald, Schnee und Landschaft, Bern, Switzerland (in German).

Laugier, F. (2007). "Design and construction of the first Piano Key Weir spillway at Goulours Dam." Int. J. Hydropower Dams, 13(5), 94-100.

Leite Ribeiro, M., Pfister, M., Schleiss, A. J., and Boillat, J.-L. (2012a). "Hydraulic design of A-type Piano Key Weirs." J. Hydraulic Res., 50(4), 400-408.

Leite Ribeiro, M., Bieri, M., Boillat, J.-L., Schleiss, A. J., Singhal, G., and Sharma, N. (2012b). "Discharge capacity of Piano Key Weirs." J. Hydraul. Eng., 138(2), 199-203.

Lempérière, F., Vigny, J. P., and Ouamane, A. (2011). "General comments on Labyrinth and Piano Key Weirs: The past and present." Proc., Intl.
Conf. Labyrinth and Piano Key Weirs, Liège B, CRC Press, Boca Raton, FL, 17-24.

Machiels, O., Erpicum, S., Dewals, B. J., Archambeau, P., and Pirotton, M. (2011). "Experimental observation of flow characteristics over a Piano Key Weir.” J. Hydraulic Res., 49(3), 359-366.

Melville, B. W., and Dongol, D. M. (1992). "Bridge pier scour with debris accumulation.” J. Hydraul. Eng., 118(9), 1306-1310.

Novak, P., Guinot, V., Jeffrey, A., and Reeve, D. E. (2010). Hydraulic modelling: An introduction, Spon Press, London.

Ouamane, A., and Lempérière, F. (2006). "Design of a new economic shape of weir." Proc., Int. Symp. Dams in the Societies of the 21st Century, Taylor \& Francis, London, UK, 463-470.

Pagliara, S., and Carnacina, I. (2010). "Temporal scour evolution at bridge piers: effect of wood debris roughness and porosity." J. Hydraulic Res., 48(1), 3-13.

Pralong, J., et al. (2011). "A naming convention for the piano key weirs geometrical parameters." Proc., Int. Conf. Labyrinth and Piano Key Weirs, Liège B, CRC Press, Boca Raton, FL, 271-278.

Schmocker, L., and Hager, W. H. (2011). "Probability of drift blockage at bridge decks." J. Hydraul. Eng., 137(4), 470-479. 